\title{
Prevalencia de prematuridad y factores de riesgo asociados en recién nacidos, ingresados en el área de neonatología del Hospital Vicente Corral Moscoso, 2011- 2015
}

Prevalence of prematurity and risk factors in newborns admitted to the neonatology area, Hospital Vicente Corral Moscoso, 2011-2015

Fabián Alejandro Suin Guaraca. ${ }^{1}$, Katiuska Vanessa Suriaga Ramírez. ${ }^{2}$, Pamela Elisa Cordero Torres. ${ }^{3}$ \& Héctor Fabián Siguencia Astudillo. ${ }^{4}$

DOI: $\underline{\text { https://doi.org/10.33262/anatomiadigital.v3i2.1..1255 }}$

\begin{abstract}
.
Prematurity, worldwide, is the leading cause of neonatal mortality and morbidity, making it a real challenge for the Ecuadorian health system. The main objective of the research is to establish the prevalence of prematurity and its associated factors, in the Neonatology area of the Vicente Corral Moscoso Hospital, between 2011-2015, applying an analytical, crosssectional observational methodology, reviewing a number of 447 clinical records

\section{Resumen.}

La prematuridad, a nivel mundial constituye la primera causa de mortalidad y morbilidad neonatal, siendo por ello un verdadero reto para el sistema de salud Ecuatoriano, el objetivo principal de la investigación es el establecimiento de la prevalencia de prematuridad y sus factores asociados, en el área de Neonatología del Hospital Vicente Corral Moscoso, entre los años 2011-2015, aplicando una metodología analística, transversal observacional, revisando un

\footnotetext{
${ }^{1}$ Red Complementaria de Salud, Cuenca Ecuador. alejandrosuin@ @otmail.com, ORCID ID 0000-0002-80930413.

${ }^{2}$ Red Complementaria de Salud, Cuenca Ecuador, katulina11@ gmail.com, ORCID ID 0000-0001-6158-3092

${ }^{3}$ Unidad Provincial del Seguro Social Campesino Morona Santiago Ecuador, pamitacordero_26@ hotmail.com, ORCID ID 0000-0002-3672-1374

${ }^{4}$ Tratante área de Neonatología Hospital Vicente corral Moscoso. fabian.siguencia@ucuenca.edu.ec, ORCID ID 0000-0001-5719-0878
} 
of newborns, who met the inclusion criteria, statistically analyzing, reporting Odds ratio, with a confidence interval of (95\%) and accepting the pi value, the relevant bioethical foundations were applied, within the results it is given that the prevalence of prematurity was $(29.4 \%)$, the percentage of adolescent mothers was ( $30.3 \%),(56.1 \%)$ have secondary education, (36.4\%) are married and $(72.7 \%)$ spend the night in urban areas. The predominant sex of premature infants was male with $(52.3 \%)$, $(70.5 \%)$ were late preterm infants and $79.5 \%$ were small for gestational age. The risk factors that show a statistical variation relationship were: age younger than 19 years old and older than 35 years old (OR: 1.71; CI: $1.12-2.59$ p: 0.01), hypertensive disorders (OR: 1.81; CI: 1.08-3.03 p: 0.02 ), intrauterine growth restriction (OR: 4.89 CI: 3.17-7.55 p: 0.000), and multiple pregnancy (OR: 2.79; CI: $1.45-5.34$ p: 0.001 ), concluding that the prevalence of prematurity was high, a relationship was found statistically significant with: age, hypertensive disorders, intrauterine growth retardation and multiple pregnancy

Keywords: Prematurity, Risk, Multiple pregnancy, neonatology número de 447 historias clínicas de neonatos, los cuales cumplieron los criterios de inclusión, analizando estadísticamente, reportándose Odds ratio, con un intervalo de confianza del (95\%) y aceptando el pi value, se aplicaron los fundamentos bioéticos pertinentes, dentro de los resultados se da que la prevalencia de prematuridad fue del $(29,4 \%)$, el porcentaje de madres adolescentes fue de $(30,3 \%)$, el $(56,1 \%)$ posee instrucción secundaria, $(36,4 \%)$ son casadas y un $(72,7 \%)$ pernoctan en zonas urbanas. El sexo predominante de los prematuros fue masculino con $(52,3 \%)$, el $(70,5 \%)$ fueron prematuros tardíos y el 79,5\% pequeños para la edad gestacional. Los factores de riesgo que muestran relación variación estadística fueron: edad menor a 19 años y mayor 35 años ( OR:1.71 ; IC: 1.12-2.59 p: 0.01), trastornos hipertensivos (OR:1.81; IC: 1.08-3.03 p: $0.02)$, restricción de crecimiento intrauterino (OR:4.89 IC:3.17-7.55 p: 0.000), y embarazo múltiple (OR: 2.79; IC: 1.45-5.34 p: 0.001), concluyendo que la prevalencia de prematuridad fue elevada, se encontró relación estadísticamente significativa con: edad, trastornos hipertensivos, retardo del crecimiento intrauterino y embarazo múltiple

Palabras claves: Prematuridad, Riesgo, Embarazo múltiple, neonatología

\section{Introducción.}

La prematuridad comprende un problema de salud pública, que se ha mantenido a través de los tiempos, afectando a los recién nacidos, de menos de 37 semanas completas de gestación contadas a partir del último día del periodo menstrual, esta problemática eleva el riesgo de adaptación en el recién nacido a la vida extrauterina, debido a la inmadurez anatomofisiológica, desembocando múltiples complicaciones respiratorias infecciosas y neurológicas, al momento del Nacimiento aumentando la morbilidad infantil. (Salgue et al. 2009) 
Mendoza, et al. (2016) y De Sousa et al. (2017) manifiestan que el resultado de la prematuridad, genera grandes costos económicos y sociales, vinculado por algunos factores demográficos y obstétricos como son el bajo nivel socioeconómico la edad materna el antecedente de parto prematuro el sangrado vaginal en el segundo trimestre. En el Ecuador el problema de mortalidad neonatal aporta cerca del 60\%, en menores de un año y alrededor del $70 \%$ en menores de menos de 28 días de nacidos, los cuales cuentan con bajo peso dentro de la edad gestacional representada por el $1 \%$ de nacimientos, contribuyendo con un $40 \%$ de mortalidad infantiles (Afonso, 2017)

En la actualidad se requiere contar con información actualizada de la prevalencia de nacimientos prematuros y los factores de riesgos asociados, por todo lo que significa el cuidado y atención de un niño prematuro y su impacto sobre la vida de sus familiares y la sociedad dentro de los aspectos sociales psicológicos económicos y de salud (Mendoza et al., 2015)

El manejo de la prematuridad constituye un verdadero desafío para las casas de salud del país debido a que el recién nacido prematuro por todas las condiciones presentadas necesita cuidados especializados los cuales requieren un amplio conocimiento y la intervención mediante múltiples procedimientos para proveer una aproximación razonable en lo que es el diagnóstico prevención evaluación y tratamiento con la finalidad de que se contribuye a disminuir la morbilidad neonatal derivado de la prematurez (Guzmán y María 2016).

Lo que se busca dentro de la investigación como objetivo general es establecer una relación entre prematuridad y los factores de riesgo asociados de los recién nacidos ingresados en el área de neonatología del hospital Vicente Corral moscoso entre los años 2011 al 2015, y los objetivos específicos están: en determinar las características sociodemográficas de la población en estudio caracterizar a los recién nacidos prematuros según el sexo peso al nacer y edad de gestación por Ballard y establecer la asociación entre prematuridad y factores de riesgo como son: ruptura prematura de membranas, trastornos hipertensivos, retardo del crecimiento intrauterino, embarazo múltiple, IVU y controles prenatales insuficientes. Para realizar el diagnóstico y el cálculo de las semanas de gestación se utiliza la última fecha de menstruación la ecografía y al momento del nacimiento se aplica la prueba de Ballard, el mismo que constituye en un instrumento de fácil acceso valorando una serie de características físicas y neurológicas colocando como resultado en cada casilla según la edad gestacional la investigación de estas características para poder ser evaluado al resultado subjetivo.

Consecuencias de la prematuridad, Romero, Carrera y Rodríguez (2012), expresan que al nacer en prematuridad trae consigo una serie de trastornos cuya severidad dependerá del número de semanas de gestación que se hayan cumplido por lo que un recién nacido prematuro presenta diversas alteraciones propias de su inmadurez como la membrana hialina taquipnea hipoglucemia hiperbilirrubinemia alteraciones nutricionales y deshidratación. (Schonhault, Perez y Astudillo 2012) El desarrollo cognitivo y de la Salud Mental con la 
prematuridad causando un progreso anormal déficit de atención trastornos emocionales y del comportamiento afectan también al rendimiento académico, relación inversamente proporcional a la edad del gestacional del nacimiento (Schonhault, Perez y Astudillo 2012).

A nivel del sistema nervioso central presenta un proceso madurativo en el último trimestre de gestación y en el primer mes luego del nacimiento con esta problemática el prematuro presentar alteraciones estructurales manifestándose con una disminución en la sustancia gris cortical, provocando trastornos a nivel cognitivo dificultades en razón de la memoria la atención el razonamiento el aprendizaje la conducta y el rendimiento escolar se conoce además que dichos problemas conductuales pueden acarrear conductas antisociales ansiedad y depresión en edades adultas (Valdes et al (2015)

Factores asociados a la prematuridad: están la edad materna, los controles prenatales, rotura prematura de membranas, IVU, trastornos hipertensivos del embarazo, restricción del crecimiento intrauterino y embarazo múltiple.

Edad materna, Rodríguez y Rossell (2013) expresan que la edad en que una mujer se convierte en madre pasando por el proceso del cuidado de su embarazo y en el producto de su gestación. Mendoza et al., (2015), concluyen en su estudio que el ser madres menores de 19 años hubo un odds radio de 1,57 , relacionando con la prematuridad

Controles prenatales, el Ministerio de Salud Pública (2015), Reyes y Khiara (2017) expresan, que un control adecuado durante todo el embarazo resulta de Vital importancia para que esté culmina de la mejor manera y en el tiempo determinado considerando 13 controles óptimos gestacionales y un mínimo de 5, la disminución de los mismos podría estar relacionado con un nacimiento prematuro. Mendoza et al., (2015), demostraron que una mala adherencia a los controles prenatales actúo como riesgo de prematuridad

Rotura prematura de membranas, el Ministerio de Salud Pública (2015), Schonhault, Perez y Astudillo (2012) expresan que la rotura de membranas cori amnióticas, Se presentan antes de que se inicie la labor del parto estimulado por varios problemas como son los inflamatorios e infeccioso presentando riesgo de morbilidad en la madre y el niño desencadenando Además del parto prematuro.

Infección de vías urinarias (IVU), Varios autores, entre estos Guadalupe y Oshiro (2015) demuestran la relación entre la infección de vías urinarias con la prematuridad Al igual que presenta relación directa con la infección del tracto urinario.

Trastornos hipertensivos del embarazo, la preclamsia, eclampsia y síndrome de HELLP, Son reconocidas de esta problemática amenazando la vida de la madre razón por la cual se debe interrumpir el inmediato el embarazo inmediatamente llegando a ser una causa de nacimiento prematuro. (Silva et al y García et al, 2016). 
Mendoza et al., (2016) encontró una relación estadística significativa entre los trastornos hipertensivos y la prematuridad Claros y Mendoza (2015) menciona entre sus investigaciones que encontró una relación entre prematuridad y preeclampsia eclampsia.

Restricción del crecimiento intrauterino, está influenciada por alteraciones que afectan directamente a la circulación y actividad de la placenta repercutiendo en el desarrollo y crecimiento fetal y en la condición de salud de la madre pudiendo desencadenar un nacimiento prematuro (Pimiento y Beltrán 2015).

Mendoza et al., (2015) menciona en su en su estudio la relación entre esta condición y la prematuridad Por otra parte Arteaga et al., (2013) manifiesta en sus estudios que atendió a 313 neonatos diagnosticados con prematuridad de quién es un $14.7 \%$ fueron diagnosticados con restricción del crecimiento intrauterino.

Embarazo múltiple, El contar con dos o más fetos durante el período de gestación es un factor determinante relacionado con la prematuridad la cual se puede presentar en un $50 \%$ de estos embarazos antes de las 27 semanas ya es un $30 \%$ menos de 32 semanas en nacimientos triples, Leal et al., (2016) y Ahumada y Barrios (2016) demostraron que el embarazo múltiple, está relacionado con la prematuridad y el embarazo gemelar.

\section{Metodología.}

La investigación este tipo analítica, visual de prevalencia y corte transversal, realizada en la provincia de Azuay, correspondiente a la zona 6 de salud del Ecuador dentro el área de neonatología del Hospital Vicente Corral Moscoso. La unidad de análisis corresponde a los neonatos ingresados Durante los años 2011 al 2015 en el área de neonatología constituida por universo de estudio de 2698 en neonatos. Para realizar el cálculo muestral se utilizó como base los supuestos de prevalencia de prematuridad del $11.4 \%$, expresado por Marín metal (2006), que posee una confiabilidad del $97 \%$ un error del $3 \%$ obteniendo una muestra mínima de 447 neonatos Posteriormente se estratifica por años quedando constituida como podemos observar en la tabla N.1

Tabla 1. Estratificación por años de los neonatos

\begin{tabular}{llll}
\hline Años & Población & Porcentaje & Muestra \\
\hline $\mathbf{2 0 1 1}$ & 430 & 16 & 72 \\
\hline $\mathbf{2 0 1 2}$ & 532 & 20 & 89 \\
\hline $\mathbf{2 0 1 3}$ & 541 & 20 & 89 \\
\hline $\mathbf{2 0 1 4}$ & 678 & 25 & 112 \\
\hline $\mathbf{2 0 1 5}$ & 517 & 19 & 85 \\
\hline Total & 2698 & 100 & 447 \\
\hline
\end{tabular}

Fuente: Elaboración propia. 
Dentro de Los criterios de inclusión se basaron las historias clínicas de neonatos ingresados en el área de neonatología del hospital Vicente Corral moscoso entre el período comprendido del año 2011 al año 2015, asimismo los criterios de exclusión nos demuestran que las historias clínicas con datos incompletos no entran dentro del análisis investigativo.

Las variables utilizadas en el estudio comprenden variables sociodemográficas como son la etnia edad instrucción estado civil y residencia permanente de la madre. La variable dependiente comprende la prematuridad, las variables independientes están ruptura prematura de membranas, retardo del crecimiento intrauterino, número de controles prenatales, trastornos hipertensivos de la gestación, periodo intergenésico, IVU, y embarazo múltiple

Se valoró y cuantificó mediante el método analítico, visual, la técnica desarrollada se dio mediante la recolección de datos utilizando un cuestionario con las variables evaluadas y definidas por los investigadores. Dentro de los instrumentos están la información recolectada mediante el formulario elaborado por los investigadores constando de varias secciones la primera concerniente a los datos sociodemográficos, la segunda y tercera sección recopilo datos sobre los factores asociados propuestos en esta investigación.

Dentro de los aspectos éticos, la información se recopiló con total confidencialidad, contando abiertamente con la autorización del comité de bioética de la Universidad de Cuenca, así como también de las autoridades competentes del hospital, procediendo a codificar los formularios con una numeración de tres dígitos iniciados desde el 001 hasta el 447.

Dentro de los procedimientos implementados está la autorización vigente luego de la aprobación del protocolo por parte del comité de ética de la Universidad de Cuenca aprobada y validada mediante un oficio de gestión documental contando con una supervisión del Dr. Fabián Sigüenza en calidad de director.

El análisis y tabulación de datos se realizó mediante el programa de Microsoft Excel para luego ser analizados en el paquete estadístico SPSS v. 15, presentando los resultados en tablas personalizadas, para las variables cualitativas se utilizó frecuencias y porcentajes (f \%) y para las variables cuantitativas que corresponde a la media y desviación estándar ( $\bar{X}$, DS). Aplicando la prueba de chi cuadrado para determinar la asociación entre la prematuridad y los factores de riesgo con un intervalo de confianza del 95\% aceptando así la hipótesis alterna si el valor de pi valor resulta inferior al 0.05

\section{Resultados.}

EN la tabla 2, se presenta la distribución de 447 recién nacidos del área de neonatología del Hospital Vicente Corral Moscoso, según las características sociodemográficas del año 20112015 
Tabla 2. Distribución recién nacidos HVCM

\begin{tabular}{|c|c|c|c|}
\hline & \\
\hline & & Frecuencia & Porcentaje \\
\hline \multirow[t]{6}{*}{ EDAD } & $=\mathrm{o}<19$ años & 110 & 24.6 \\
\hline & 20 a 24 años & 137 & 30.6 \\
\hline & 25 a 29 años & 87 & 19.5 \\
\hline & 30 a 34 años & 63 & 14.1 \\
\hline & 35 años o más & 50 & 11.2 \\
\hline & Total & 447 & 100 \\
\hline \multirow[t]{6}{*}{ ETNIA } & Blanca & 1 & 0.2 \\
\hline & Negra & 3 & 0.7 \\
\hline & Mestiza & 435 & 97.3 \\
\hline & Indígena & 7 & 1.6 \\
\hline & Otra & 1 & 0.2 \\
\hline & Total & 447 & 100 \\
\hline \multirow[t]{5}{*}{ INSTRUCCIÓN } & Primaria & 119 & 26.6 \\
\hline & Secundaria & 291 & 65.1 \\
\hline & Universitario & 32 & 7.2 \\
\hline & Otros & 5 & 1.1 \\
\hline & Total & 447 & 100 \\
\hline \multirow[t]{6}{*}{ ESTADO CIVIL } & Soltera & 110 & 24.6 \\
\hline & Casada & 179 & 40 \\
\hline & Divorciada & 1 & 0.2 \\
\hline & Viuda & 1 & 0.2 \\
\hline & Unión libre & 156 & 34.9 \\
\hline & Total & 447 & 100 \\
\hline \multirow[t]{3}{*}{ RESIDENCIA } & Urbana & 359 & 80.3 \\
\hline & Rural & 88 & 19.7 \\
\hline & Total & 447 & 100 \\
\hline
\end{tabular}

Fuente: Hospital Vicente Corral Moscoso.

La tabla 2, muestra datos sociodemográficos donde la mayor parte de las madres de los neonatos están en un Rango de edad de 20 a 24 años con el 30.6\% seguidas de las adolescentes con un $24.6 \%$ y las mujeres de 25 a 29 años presentaron una frecuencia del 19.5\% . El $97.3 \%$ auto identificándose como mestizas el $1.6 \%$ como indígenas el $65.1 \%$ culminó sus estudios secundarios el $26.6 \%$ la primaria un $40 \%$ Indicó que su estado civil casada un $34.9 \%$ unión libre el $24.6 \%$ soltera la residencia urbana predominó con el $80.3 \%$ y la residencia rural con $19.7 \%$ 
Tabla 3. Prevalencia de prematuridad en 447 pacientes del Área de Neonatología del Hospital Vicente Corral Moscoso. 2011-2015.

\begin{tabular}{llll}
\hline \multirow{2}{*}{ PREMATURIDAD } & & Frecuencia & Porcentaje \\
\cline { 3 - 4 } & Sí & 132 & 29.5 \\
& No & 315 & 70.5 \\
& Total & 447 & 100 \\
\hline
\end{tabular}

Fuente: Elaboración propia.

En la tabla número 3 observamos que la prevalencia de prematuridad en el área de neonatología del hospital Vicente Corral moscoso está entre los años 2011 a 2015 con un $29.5 \%$, concordando con cifras expuestas por Leal et al.(2016), quienes concluyeron una prevalencia de $11.5 \%$ en su estudio realizado en gestantes de una población brasileña; al igual que Mendoza Tascón et al., en Colombia quienes reportaron un 11,4\% (9), a su vez Azevedo et at., presentaron cifras inferiores de prematuridad con un $8 \%$ en Minas GeraisBrasil, estudio realizado por Guimarães et al. (2008)

Tabla 4. Distribución de 132 recién nacidos prematuros Área de Neonatología del Hospital Vicente Corral Moscoso. 2011-2015, características sociodemográficas.

\begin{tabular}{llll}
\hline & & \multicolumn{2}{l}{ Prematuridad } \\
\cline { 3 - 4 } EDAD & Frecuencia & Porcentaje \\
\cline { 3 - 4 } & 20 a 24 años & 40 & 30.3 \\
& 25 a 29 años & 19 & 27.3 \\
30 a 34 años & 18 & 14.4 \\
& 35 años o más & 19 & 13.6 \\
& Total & 132 & 14.4 \\
\hline ETNIA & Negra & 1 & 0.8 \\
& Mestiza & 125 & 94.7 \\
& Indígena & 6 & 4.5 \\
& Total & 132 & 100 \\
\hline INSTRUCCIÓN & Primaria & 46 & 34.8 \\
& Secundaria & 74 & 56.1 \\
& Universitario & 10 & 7.6 \\
& Otros & 2 & 1.5 \\
& Total & 132 & 100 \\
\hline ESTADO CIVIL & Soltera & 43 & 32.6 \\
& Casada & 48 & 36.4 \\
& Unión libre & 41 & 31.1 \\
& Total & 132 & 100 \\
\hline
\end{tabular}




\begin{tabular}{llll} 
RESIDENCIA & Urbana & 96 & 72.7 \\
& Rural & 36 & 27.3 \\
& Total & 132 & 100 \\
\hline
\end{tabular}

Fuente: Elaboración propia.

De acuerdo con las características sociodemográficas las madres que tuvieron recién nacidos prematuros comprenden un $30.3 \%$ las cuales fueron adolescentes el $27.3 \%$ sus edades oscilan entre 20 a 24 años el 94.7\% identificándose con la etnia mestiza el 56.1\% fueron distribución secundaria el $36.4 \%$ casadas el $32.6 \%$ solteras y el $72.7 \%$ tienen residencia urbana versus un $27.3 \%$ residencia en áreas rurales.

Tabla 5. Distribución de 132 recién nacidos prematuros, recién nacidos prematuros Área de Neonatología del Hospital Vicente Corral Moscoso. 2011-2015, según sexo, edad gestacional y peso

\begin{tabular}{llll}
\hline & & \multicolumn{2}{l}{ Recién Nacidos Prematuros } \\
\cline { 3 - 4 } SEXO & Hombre & 69 & Precuencia \\
& Mujer & 63 & 52.3 \\
& Total & 132 & 47.7 \\
& Muy prematuros & 15 & 100 \\
\hline CLASIFICACIÓN & 24 & 11.4 \\
POR EDAD & Prematuros moderados & 93 & 18.2 \\
GESTACIONAL & Prematuros tardíos & 132 & 70.5 \\
& Total & 105 & 100 \\
\hline PESO CON & Pequeño para la edad gestacional & & 79.5 \\
RELACIÓN A EDAD & & 26 & 19.7 \\
GESTACIONAL & Adecuado para la edad gestacional & 26 & 0.8 \\
& Grande para la edad gestacional & 1 & 100 \\
\hline
\end{tabular}

Fuente: Elaboración propia.

En la tabla número 5 evidenciamos la distribución de los recién nacidos prematuros de acuerdo al sexo donde predominan los neonatos varones con un 52.3\% los neonatos femeninos $47.7 \%$ en cuanto a la edad gestacional los prematuros tardíos comprenden un $70.5 \%$, los moderados un $18.2 \%$ y el $11.4 \%$ fue para los muy prematuros, en cuanto a la relación del peso con edad gestacional el $79.5 \%$, fue pequeño y el $19.7 \%$, adecuado para la edad gestacional. 
Tabla 6. Distribución de 447 recién nacidos del Área de Neonatología del Hospital Vicente Corral Moscoso según factores asociados a prematuridad. 2011-2015.

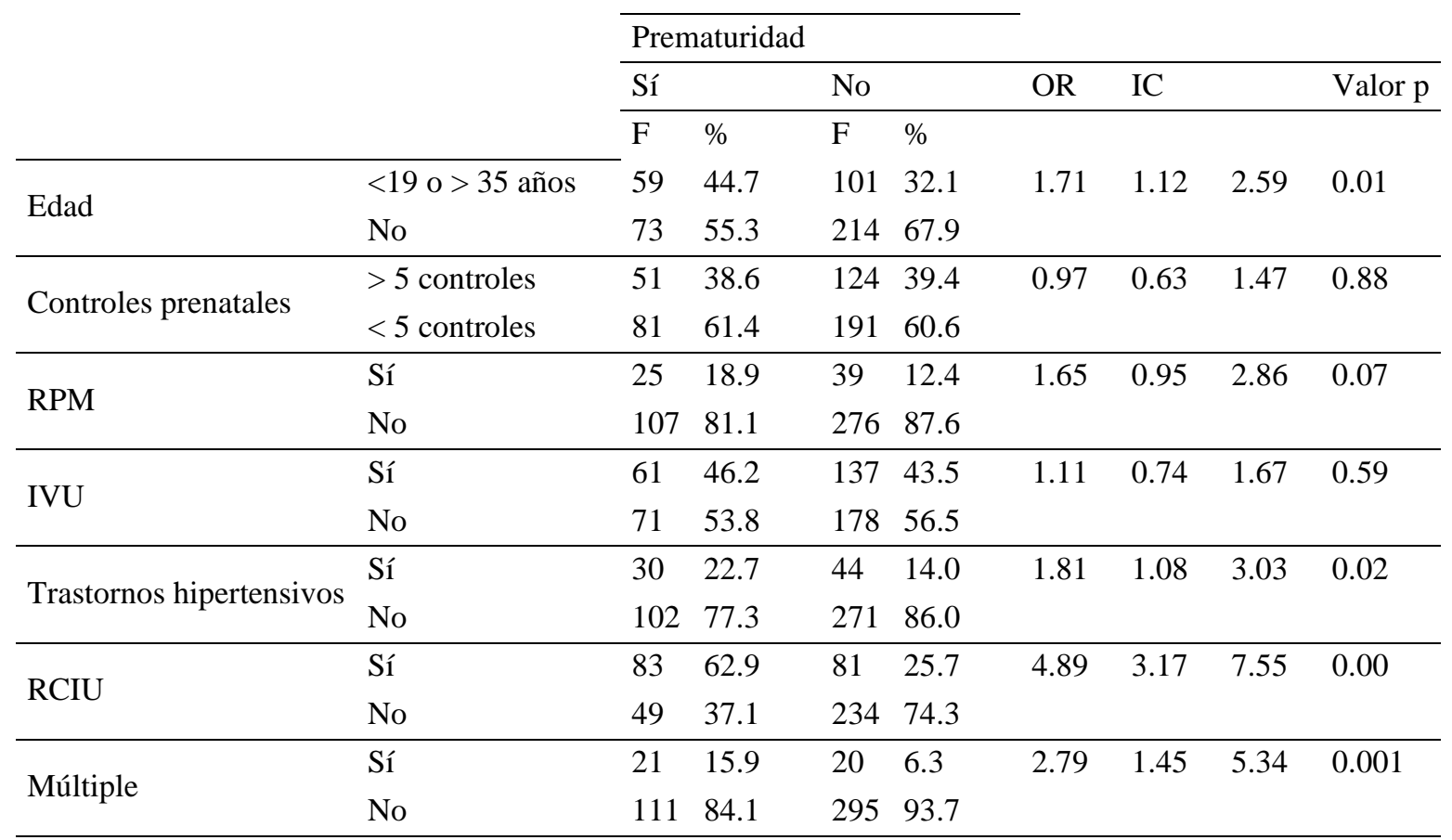

Fuente: Elaboración propia.

Con respecto de la relación de factores de riesgo asociados, se concluyó que: la edad menor a 19 años y mayor a 35 (OR:1.71 ; IC: $1.12-2.59$ p: 0.01 ), los trastornos hipertensivos (OR:1.81; IC: 1.08-3.03 p:0.02 ), la restricción de crecimiento intrauterino (OR:4.89 IC:3.177.55 p: 0.000), y el embarazo múltiple (OR: 2.79; IC: 1.45-5.34 p: 0.001), los cuales obtuvieron una relación estadísticamente significativa con prematuridad. Sin embargo, no se encontró esta relación con variables como: número de controles prenatales, ruptura prematura de membranas, IVU.

En cuanto al aspecto de la edad materna, de los datos resultantes la relación entre la Edad materna y prematuridad de las madres menores de 19 años y mayores de 35 años tuvieron una relación estadísticamente significativa (OR: 1.71 IC: 1.12-2-59 p: $<0,001$ ), concordando con Azevedo et al (2015), donde madres menores de 15 años, fueron consideradas como grupo de riesgo presentado un (OR 1.22 IC. 95\% 1.01-1.49 p: <0.029), Mendoza et al (2016) menciono la razón de momios de 1.57 IC: 1.29-1.87 p <0.001, cuyos grupos de edad entre 13 y 19 años y madres entre 14 a 19 y 35 a 50 años presentaron un OR de 4.2 IC 2.77-6.40 p $<0.001$. 
El aspecto de controles prenatales, se encontró una relación estadísticamente significativa con el numero de controles prenatales (menores a 5) (OR de 0.97, IC de 0.63-1.47 p=0.88), mientras que Mendoza et al (2016)., evidenciaron que la mala adherencia a controles prenatales presentó un OR 1.55 IC 1.29-1.87, p: < 0.001.

En cuanto al retardo del crecimiento intrauterino, Mendoza et al (2016), demostrando mediante estudios de casos y controles en una población de 11811 madres gestantes Qué es la restricción de crecimiento intrauterino se relaciona directamente con factor para el nacimiento prematuro (OR 1.91 IC 1.09-3.32 $\mathrm{p}<0.023$ ), empatizando con nuestra investigación la cual dio como resultado el OR 4.89 IC 3.17-7.55 p 0.000, quedando comprobado estadísticamente que va en asocio con la prematuridad.

El embarazo múltiple analizado con los estudios de Leal et al (2016), En Brasil con 23940 madres indicaron que el embarazo múltiple fue un factor fuerte asociado a la prematuridad, (OR 16.42 IC 10.56-25.53). En concordancia con nuestra investigación, puesto que encontramos una relación estadísticamente significativa con embarazo múltiple y prematuridad (OR 2.79 IC 1.45-5.34 p 0.001)

En la ruptura prematura de membranas, no se encuentra relación entre RPM y prematuros (OR 1.65 IC 0.95-2.86 p 0.07), contrariamente con los estudios realizados por Rodríguez, Ramos y Hernández (2013) encontrando relación estadísticamente significativa entre la variable a investigar y el parto prematuro (OR 1.67 IC 1.25-2.24 p < 0.0005).

Los trastornos hipertensivos de la gestación, en la población de estudio dan resultados de OR 1.81 IC $95 \%$ 1.08-3.03 p 0.02 en relación con nacer prematuro, estudios que concuerdan con Ahumada y Barrios (2016) en los estudios realizados en la ciudad de Lima, Perú, dentro de los estudios de caso y controles reveló que la preeclampsia es realmente un factor asociado a prematuridad (OR $1.9 \mathrm{p}<0.005)$.

La infección de vías urinarias en nuestra investigación, si las madres padecen una infección de tracto urinario en la gestación no se determinó como un factor relacionado a parto prematuro (OR 1.11 IC 0.74-1.67 p 0.59), en contrapunto con la investigación realizada por Rodríguez et al (2013), si encontraron relación estadísticamente significativa entre la infección de vías urinarias y la prematuridad (OR 3.63 IC al 9\%\% 2.67-4.92 p < 0.0001)

\section{Conclusiones.}

- La mayoría de las madres del estudio, se auto consideraron como mestizas, ubicándose en el rango de edad entre 20 a 24 años, seguidas de las madres adolescentes, además se conoció qué más de la mitad de las mujeres poseen instrucción secundaria y su residencia está en la zona urbana.

- Dentro del análisis del estudio se conoce que la prevalencia de prematuridad en los neonatos fue de $29.5 \%$. 
- En cuanto al análisis estadístico, no se encontraron relación con las variables: número de controles prenatales, ruptura prematura de las membranas e infección de las vías urinarias, la relación que presenta una variación estadística significativa se da con la prematuridad y factores como edad del menor a 19 años y mayores a 35 años, trastornos hipertensivos, restricción del crecimiento intrauterino y embarazo múltiple.

- Dentro de las características que se pueden Resaltar de los recién nacidos prematuros fueron que más de la mitad son de sexo masculino, en cuanto a la edad gestacional los neonatos tardíos alcanzaron un 70.5\% y en contraposición con la relación del peso con edad gestacional fue de $79.5 \%$

\section{Referencias Bibliográficas.}

Alfonso I., (2017) Prematurida de E Phda o que nos diz a Investigação. Prematur ADHD WHAT DOES Res TELL US; 8(1):119-37.

Ahumada-Barrios M., (2016) Alvarado GF. Factores de Riesgo de parto pretérmino en un hospital. :8.

Claros, D. y Mendoza, L. (2015) Impacto de los trastornos hipertensivos, la diabetes y la obesidad materna sobre el peso, la edad gestacional al nacer y la mortalidad neonatal. Rev Chil Obstet Ginecol;81(6):480-8.

De Sousa R., Rodrigues R., De Araújo J., De Melo A., Bezerra L,. (2017) Fatores De Risco Associados À Prematuridade E Baixo Peso Em Uma Maternidade Pública Em João Pessoa-Pb. RISK FACTORS Assoc Prematur LOW WEIGHT PUBLIC Matern JOÃO PESSOA-PB. ;20(2):35-40.

García N, Isabel A, Ramos B, Teresa M, Calvo García E. Síndrome HELLP en atención primaria. Med Gen Fam Ed Digit. 1 de abril de 2016;5(2):64-7.

Guadalupe-Huamán SM, Oshiro-Canashiro S. Factores de riesgo asociados a parto pretérmino en gestantes del servicio de gineco-obstetricia del Hospital María Auxiliadora durante el año 2015. Rev Fac Med Humana [Internet]. 17(1). Disponible en: http://revistas.urp.edu.pe/index.php/RFMH/article/view/746

Guimarães, E., Vieira, C., Nunes, D., Januário G., Oliveira V., Tibúrcio J.,(2008). Prevalência e fatores associados à prematuridade em Divinópolis, Minas Gerais, 2008-2011: análise do Sistema de Informações sobre Nascidos Vivos. Epidemiol E Serviços Saúde.;26(1):91-8.

Leal, M., Esteves, A., Nakamura, M., Torres, J., Theme, M., Domingues (2016) Prevalence and risk factors related to preterm birth in Brazil. Reprod Health [Internet]. octubre de 2016 [citado 14 de septiembre de 2018];13(S3). Recuperado desde: http://reproductive-health-journal.biomedcentral.com/articl es/10.1186/s12978-0160230-0, 8. 
Marín, G., Martín, J., Lliteras, G, Delgado,S., Pallás C., De la Cruz, J, (2006) Valoración del test de Ballard en la determinación de la edad gestacional. An Pediatría ;64(2):140, 5 .

Mendoza, L., Claros, D., Mendoza, L., Arias, M., Peñaranda, C., (2016) Epidemiología de la prematuridad, sus determinantes y prevención del parto prematuro. Rev Chil Obstet Ginecol. agosto de 2016;81(4):3, 30-42.

Mendoza L., Arias M., Peñaranda C., Mendoza L., Manzano S., Varela Bahena A.,(2015) Influencia de la adolescencia y su entorno en la adherencia al control prenatal e impacto sobre la prematuridad, bajo peso al nacer y mortalidad neonatal. Rev Chil Obstet Ginecol;80(4):306-15.

Ministerio de Salud Pública del Ecuador,(2015) Direccción Nacional de Normatización. Recién Nacido Pramaturo [Internet]. Disponible en: https://www.salud.gob.ec/wpcontent/uploads/2014/05/GPC-Rec\%C3\%A9n-nacido-prematuro.pdf

Ministerio de Salud Pública del Ecuador,(2015) Direccción Nacional de Normatización. Control prenatal. Guía de Práctica Clínica [Internet]. 2015. Disponible en: https://www.salud.gob.ec/guias-de-practica-clinica/

Nelson, (2013). Tratado de Pediatría. 19. a ed. España: Elsevier; 2p

Pimiento L, y Beltrán M.(2015) Restricción del crecimiento intrauterino: una aproximación al diagnóstico, seguimiento y manejo. Rev Chil Obst Ginecol. 2015;80(6):493-502

Reyes M, Khiara M. (2107) Control prenatal inadecuado como factor asociado a parto pretermino en pacientes del Hospital Regional Docente de Trujillo. Univ Priv Antenor Orrego [Internet]; Disponible en: http://repositorio.upao.edu.pe/handle/upaorep/2249

Rodríguez C, Rossell M.,(2013) Factores de riesgo asociados a la prematuridad en recién nacidos de madres adolescentes. Rev Obstet Ginecol Venez.;73(3):157-10.

Rodríguez S., Ramos R., Hernández R.. (2013)Factores de riesgo para la prematurez. Estudio de casos y controles. Ginecol Obstet México.;5

Romero, S., Carrera, S., Rodríguez, O. (2012) Morbilidad del recién nacido prematuro tardío durante su primer mes de vida comparado con el recién nacido de término. Perinatol Reprod Hum.:5.

Salge, A., Da Costa, A., Aguiar, K., Lobo, F., Xavier, M., Zatta, L.,(2009). Fatores maternos e neonatais associados à prematuridade. Matern Neonatal Factors Assoc Prematur;11(3): 642-6.

Schonhaut B, Pérez R, Astudillo D.(2012) Prematuros tardíos: un grupo de riesgo de morbilidad a corto y largo plazo. Rev Chil Pediatría; 83(3):217-23.

Silva-Ocas I, Gálvez-Olortegui J, Gálvez-Olortegui T, Tavara-Valladolid L, Fiestas-Plucker G, Chaman-Castillo J. Preeclampsia y defecto cardiaco fetal: ¿existe una asociación? Revisión de la evidencia. Rev Chil Obstet Ginecol. 2016;81(5):426-32.

Valdés, F., Fabré L., Hernández L., Garcell J., Malagón G., Fabré K.. (2015). Influencia de la prematuridad sobre el sistema nervioso en la niñez y en la adultez. :9. 
PARA CITAR EL ARTÍCULO INDEXADO.

Suin Guaraca, F. A., Suriaga Ramírez, K. V., Cordero Torres, P. E., \& Siguencia Astudillo, H. F. (2020). Prevalencia de prematuridad y factores de riesgo asociados en recién nacidos, ingresados en el área de neonatología del Hospital Vicente Corral Moscoso, 20112015. Anatomía Digital, 3(2.1.), 18-31. https://doi.org/10.33262/anatomiadigital.v3i2.1.1255

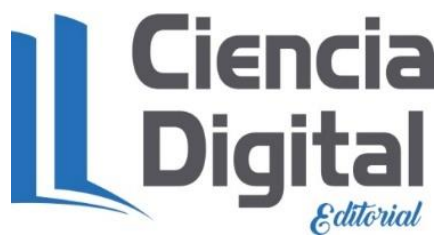

El artículo que se publica es de exclusiva responsabilidad de los autores y no necesariamente reflejan el pensamiento de la Revista Anatomía Digital.

El artículo queda en propiedad de la revista y, por tanto, su publicación parcial y/o total en otro medio tiene que ser autorizado por el director de la Revista Anatomía Digital.
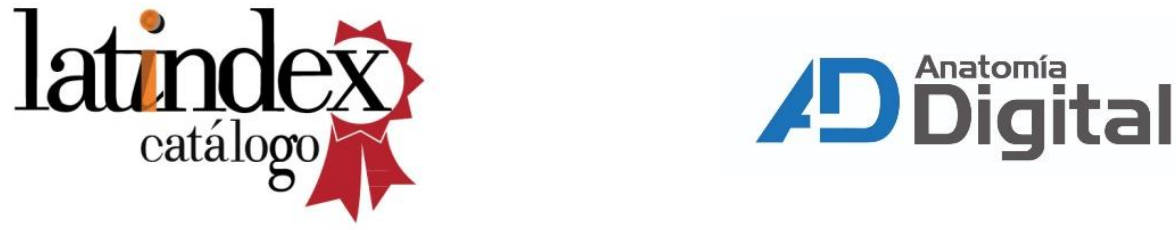